\title{
Quantum geometry from the toroidal block
}

\author{
Amir-Kian Kashani-Poor and Jan Troost \\ Laboratoire de Physique Théorique, ${ }^{1}$ École Normale Supérieure, \\ 24 rue Lhomond, 75005 Paris, France \\ E-mail: kashani@lpt.ens.fr, troost@lpt.ens.fr
}

ABSTRACT: We continue our study of the semi-classical (large central charge) expansion of the toroidal one-point conformal block in the context of the $2 \mathrm{~d} / 4 \mathrm{~d}$ correspondence. We demonstrate that the Seiberg-Witten curve and ( $\epsilon_{1}$-deformed) differential emerge naturally in conformal field theory when computing the block via null vector decoupling equations. This framework permits us to derive $\epsilon_{1}$-deformations of the conventional relations governing the prepotential. These enable us to complete the proof of the quasi-modularity of the coefficients of the conformal block in an expansion around large exchanged conformal dimension. We furthermore derive these relations from the semi-classics of exact conformal field theory quantities, such as braiding matrices and the S-move kernel. In the course of our study, we present a new proof of Matone's relation for $\mathcal{N}=2^{*}$ theory.

Keywords: Conformal and W Symmetry, Topological Strings, Supersymmetric gauge theory

ARXIV EPRINT: 1404.7378

\footnotetext{
${ }^{1}$ Unité Mixte du CNRS et de l'École Normale Supérieure associée à l'Université Pierre et Marie Curie 6, UMR 8549.
} 


\section{Contents}

1 Introduction 1

2 The toroidal one-point block $\quad 2$

2.1 The one-point function and conformal block 3

2.2 The two-point block including one degenerate insertion 3

2.3 The variable map and limits 3

3 Seiberg-Witten geometry and S-duality from null vector decoupling 4

3.1 The null vector decoupling equation 4

3.2 The amplitudes from generalized period integrals 5

3.2.1 The generalized Seiberg-Witten differential 5

3.2.2 Seiberg-Witten data and proof of the Matone relation 6

3.2.3 Beyond leading order: quantum geometry 9

3.2.4 Comparing to the proposal in [1] for the Seiberg-Witten geometry 11

3.2.5 Bohr-Sommerfeld interpretation 12

$\begin{array}{lll}3.3 & \text { Transformation properties and S-duality } & 13\end{array}$

4 Exact conformal field theory methods $\quad \mathbf{1 5}$

$\begin{array}{ll}4.1 \text { The } B \text {-monodromy from braiding } & 15\end{array}$

$\begin{array}{ll}4.2 & \text { The semi-classical S-move kernel } \\ \end{array}$

5 Conclusions $\quad 21$

$\begin{array}{ll}\text { A The function } s_{b} & 22\end{array}$

\section{Introduction}

The semi-classical limit of conformal blocks has taken on new significance with the advent of the $2 \mathrm{~d} / 4 \mathrm{~d}$ correspondence [1] between two-dimensional conformal field theory and fourdimensional $\mathcal{N}=2$ gauge theory. Holomorphic amplitudes $F^{(n, g)}$ specifying the dynamics of the gauge theory, such as the prepotential $F^{(0,0)}$, arise as coefficients in an asymptotic expansion of the blocks in this limit. Several methods of computation precede the $2 \mathrm{~d} / 4 \mathrm{~d}$ correspondence: among these, the generalized holomorphic anomaly equations compute the $F^{(n, g)}$ directly [2-5], while localization calculations yield their generating function, the Nekrasov partition function [6]. The conformal field theory perspective opens up a new avenue for the computation of the amplitudes $F^{(n, g)}$ based on null vector decoupling equations. In previous work [7,8], we demonstrated how to compute the $F^{(n, 0)}$ in this setup, for $\mathcal{N}=2^{*}$ and $N_{f}=4$ gauge theory. Here, for the case of $\mathcal{N}=2^{*}$, we will demonstrate how 
to rederive the Seiberg-Witten approach $[9,10]$ for computing the prepotential of the gauge theory within this framework, and extend these techniques to the generating function $F=$ $\sum F^{(n, 0)} \epsilon_{1}^{2 n}$. In particular, an $\epsilon_{1}$-deformed Seiberg-Witten differential will emerge naturally in this setup. As Seiberg and Witten geometrize the problem of computing the prepotential by relating it to an elliptic fibration over moduli space, and as deformation by $\epsilon_{1}$ can be interpreted as a quantum deformation from an integrable systems perspective [11], such an extension can be said to describe quantum geometry. In the course of our investigations, we will prove the quasi-modularity of the $F^{(n, 0)}$, a property we had observed experimentally in [7]. Our argument includes the first proof of Matone's relation [12] for a superconformal theory in the Seiberg-Witten framework. A proof using localization methods has appeared in [13].

A second theme of this work is extracting results we obtain from the semi-classical solution of null vector decoupling equations directly from the semi-classics of known exact relations in conformal field theory. We thus study the dual period of the $\epsilon_{1}$-deformed Seiberg-Witten differential by using braiding matrices to compute the monodromy behavior of the toroidal block, and also extract the transformation properties of the generating function $F$ under S-duality from the integral kernel implementing the S-move on the block.

Our interest in studying the amplitudes $F^{(n, g)}$ in the context of exact conformal field theory results stems from their interpretation as limits of topological string amplitudes. While the topological string partition function from a worldsheet perspective is merely a generating function for these amplitudes, it acquires a non-perturbative definition in the light of the $2 \mathrm{~d} / 4 \mathrm{~d}$ correspondence. Our hope is therefore that a careful study of how semiclassical results emerge from exact quantities in conformal field theory will help clarify non-perturbative aspects of topological string theory.

Our paper is organized as follows. In section 2, we review the toroidal one-point block of conformal field theory. In section 3, we revisit the construction of the semi-classical conformal block through a WKB solution of the null vector decoupling equation. We show how the Seiberg-Witten curve and ( $\epsilon_{1}$-deformed) differential arise naturally in this framework, allowing us to demonstrate that up to exceptional leading terms, the expansion of the logarithm of the block is in terms of quasi-modular forms. We offer a proof of Matone's relation for $\mathcal{N}=2^{*}$ theory in the course of the argument. The exact formulae for the braiding matrices and the modular S-move on the toroidal one-point block are reviewed in section 4 , and used to rederive some of the results of section 3 upon saddle point approximation. We conclude in section 5 .

\section{The toroidal one-point block}

In this section, we will briefly review the definition of the one-point conformal block of two-dimensional conformal field theory, and of the two-point conformal block that includes one degenerate insertion. These map to the instanton partition function of $\epsilon$-deformed $\mathcal{N}=2^{*}$ gauge theory [1], the latter in the presence of a surface operator [14]. 


\subsection{The one-point function and conformal block}

In a two-dimensional conformal field theory, the expectation value of an operator $V_{h_{m}}$ with conformal dimension $h_{m}$ inserted on a torus with complex structure parameter $\tau$ can be decomposed in terms of the three-point functions $C_{h_{m}, h}^{h}$ of the theory and the toroidal one-point blocks $\mathcal{F}_{h_{m}}^{h}$,

$$
\left\langle V_{h_{m}}\right\rangle_{\tau}=\sum_{h} C_{h_{m}, h}^{h}(q \bar{q})^{h-\frac{c}{24}}\left|\mathcal{F}_{h_{m}}^{h}(q)\right|^{2} .
$$

The sum here is over all the primary fields, of conformal dimensions $h$, in the spectrum of the theory. In terms of chiral vertex operators, the one-point toroidal conformal block can be represented as the trace

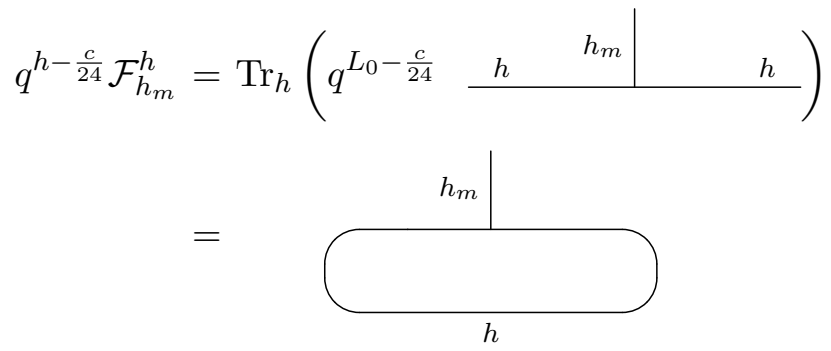

The trace is taken over the primary state $|h\rangle$ and all of its Virasoro descendants.

\subsection{The two-point block including one degenerate insertion}

To study the one-point conformal block, we will take advantage of the null vector decoupling equation satisfied by the two-point toroidal block with the additional insertion chosen to be degenerate at level two, with weight denoted $h_{(2,1)}$. In addition to the dependence on the two external weights $h_{m}$ and $h_{(2,1)}$, this block requires specifying two internal momenta $h$ and $h_{ \pm}$, in accord with the diagram

$$
\mathcal{F}_{h_{m}, h_{(2,1)}}^{h, h_{ \pm}}=\left.\underbrace{h_{m} \mid h_{ \pm}}_{h}\right|^{h_{(2,1)}}
$$

Note that due to the degenerate nature of the primary with weight $h_{(2,1)}$, this block is non-vanishing only for two choices of internal weight $h_{ \pm}$as a function of the exchanged conformal weight $h$.

\subsection{The variable map and limits}

Our calculations will take place purely within conformal field theory. By the $2 \mathrm{~d} / 4 \mathrm{~d}$ correspondence [1], the one-point toroidal block thus obtained is equal to the instanton partition 
function of $\mathcal{N}=2^{*}$ gauge theory, upon the following identification of variables:

$$
\begin{aligned}
c & =1+6 Q^{2}, & Q & =b+b^{-1}, \\
h_{m} & =\frac{Q^{2}}{4}-\frac{m^{2}}{\epsilon_{1} \epsilon_{2}}, & h & =\frac{Q^{2}}{4}-\frac{a^{2}}{\epsilon_{1} \epsilon_{2}} .
\end{aligned}
$$

The central charge $c$ of the theory is parameterized by the numbers $Q$ or $b$. In gauge theory, $a$ and $m$ are the vacuum expectation value of the adjoint vector multiplet scalar and the mass parameter of the adjoint matter multiplet respectively. In the refined topological string context [15], the $\epsilon_{i}$ deformation parameters are related to the string coupling $g_{s}^{2}=$ $\epsilon_{1} \epsilon_{2}$ and the expansion parameter $s=\left(\epsilon_{1}+\epsilon_{2}\right)^{2}$.

We will be working in the semi-classical limit of large central charge $c \rightarrow \infty(b \rightarrow 0)$ and conformal dimensions, with $h / c \rightarrow \infty$ and $h / h_{m} \rightarrow \infty$. In terms of gauge theory parameters, this corresponds to the small $\epsilon_{i}$, large vacuum expectation value limit $\epsilon_{2} / \epsilon_{1} \rightarrow$ $0, \epsilon_{1} / a \ll 1$, and $m / a \ll 1$.

\section{Seiberg-Witten geometry and S-duality from null vector decoupling}

In this section, we analyze the properties of the semi-classical solutions to the second order null vector decoupling equation satisfied by the two-point block. In particular, we identify the Seiberg-Witten data that determine the solution within conformal field theory. This permits us to prove the quasi-modularity of the expansion coefficients of the semi-classical solution, observed experimentally in [7], to all orders. In the process, we provide a proof of the Matone relation for $\mathcal{N}=2^{*}$ theory.

\subsection{The null vector decoupling equation}

Our principal strategy for computing the one-point toroidal block and determining its transformation properties is, as in [7, 8, 16-18], to consider an additional, second order degenerate insertion on the torus. Due to the degeneracy of the insertion, the resulting two-point function satisfies a differential equation, the null vector decoupling equation, which upon rescaling of the two-point function,

$$
\Psi(z \mid \tau)=\theta_{1}(z \mid \tau)^{-\frac{b^{2}}{2}} \eta(\tau)^{-2\left(h_{m}-b^{2}-1\right)} Z\left\langle V_{h_{(2,1)}}(z) V_{h_{m}}(0)\right\rangle_{\tau},
$$

takes the simple form $[7,18]$

$$
\left[-\frac{1}{b^{2}} \partial_{z}^{2}-\left(\frac{1}{4 b^{2}}-\frac{m^{2}}{\epsilon_{1} \epsilon_{2}}\right) \wp(z)\right] \Psi(z \mid \tau)=2 \pi i \partial_{\tau} \Psi(z \mid \tau)
$$

The function $\wp$ is the Weierstrass $\wp$-function associated to the torus of periods $(1, \tau)$ on which the conformal field theory lives, and the function $Z$ is the partition function. Imposing the monodromy $[7,19]$

$$
\Psi(z+1)=e^{ \pm 2 \pi i \frac{a}{\epsilon_{1}}} \Psi(z)
$$


on the solution to the differential equation permits us to project onto the conformal block $\mathcal{F}_{h_{m}, h_{(2,1)}}^{h, h_{ \pm}}$, in the notation of (2.4). The analysis up to this point is exact. To extract the one-point conformal block of interest from the rescaled two-point function $\Psi$, we need to take the semi-classical $\epsilon_{2} / \epsilon_{1} \rightarrow 0$ limit, in which the degenerate insertion becomes light and its contribution to the conformal block is multiplicative (as can be seen in a semi-classical analysis of Liouville theory (see e.g. [20])). This motivates the factorized ansatz

$$
\Psi(z \mid \tau)=\exp \left[\frac{1}{\epsilon_{1} \epsilon_{2}} \mathcal{F}(\tau)+\frac{1}{\epsilon_{1}} \mathcal{W}(z \mid \tau)\right]
$$

with functions $\mathcal{F}$ and $\mathcal{W}$ that are independent of $\epsilon_{2}$. In terms of this ansatz, the one-point block is given by

$$
\left.\lim _{\epsilon_{2} \rightarrow 0} Z\left\langle V_{h_{m}}\right\rangle\right|_{h}=\exp \left[\frac{1}{\epsilon_{1} \epsilon_{2}}\left(\mathcal{F}+2\left(-m^{2}+\frac{\epsilon_{1}^{2}}{4}\right) \log \eta\right)\right] .
$$

The null vector decoupling equation (3.2) evaluated on the ansatz (3.4) yields the equation

$$
-\frac{1}{\epsilon_{1}} \mathcal{W}^{\prime \prime}(z \mid \tau)-\frac{1}{\epsilon_{1}^{2}} \mathcal{W}^{\prime}(z \mid \tau)^{2}+\left(\frac{1}{\epsilon_{1}^{2}} m^{2}-\frac{1}{4}\right) \wp(z)=(2 \pi i)^{2} \frac{1}{\epsilon_{1}^{2}} q \partial_{q} \mathcal{F}(\tau)+\frac{\epsilon_{2}}{\epsilon_{1}^{2}} 2 \pi i \partial_{\tau} \mathcal{W}(z \mid \tau),
$$

while the boundary condition (3.3) projecting onto the desired conformal block maps to the condition

$$
\mathcal{W}(z+1)-\mathcal{W}(z)= \pm 2 \pi i a
$$

In [7], we solved this differential equation (dropping the linear term in $\epsilon_{2}$ ) in a formal $\epsilon_{1}$-expansion of $\mathcal{F}$ and $\mathcal{W}$

$$
\mathcal{F}(\tau)=\sum_{n=0}^{\infty} \mathcal{F}_{n}(\tau) \epsilon_{1}^{n}, \quad \mathcal{W}(z \mid \tau)=\sum_{n=0}^{\infty} \mathcal{W}_{n}(z \mid \tau) \epsilon_{1}^{n},
$$

and demonstrated to a given order that the coefficients $\mathcal{F}_{n}$ of the non-convergent expansion reproduce the modular results obtained from the holomorphic anomaly equations [5] and localization calculations [21, 22],

$$
F^{(n, 0)}=\mathcal{F}_{2 n}
$$

\subsection{The amplitudes from generalized period integrals}

\subsubsection{The generalized Seiberg-Witten differential}

Combining equations (3.6) and (3.7) yields the equation

$$
\int_{0}^{1} \sqrt{m^{2} \wp-(2 \pi i)^{2} q \partial_{q} \mathcal{F}-\epsilon_{1} \mathcal{W}^{\prime \prime}-\epsilon_{1}^{2} \frac{\wp}{4}} d z= \pm 2 \pi i a .
$$

Given that the variable $a$ maps to the vacuum expectation value of the adjoint scalar field in $\mathcal{N}=2^{*}$ gauge theory, we wish to interpret

$$
\lambda:=\sqrt{m^{2} \wp-(2 \pi i)^{2} q \partial_{q} \mathcal{F}-\epsilon_{1} \mathcal{W}^{\prime \prime}-\epsilon_{1}^{2} \frac{\wp}{4}} d z
$$


as a generalized $\epsilon_{1}$-dependent Seiberg-Witten differential. We will justify this interpretation by demonstrating that the $B$-cycle period of $\lambda$ computes the $a$-derivative of the generalized prepotential $\mathcal{F}$,

$$
2 \pi i a_{D}:=\oint_{B} \lambda=-\frac{1}{2} \frac{\partial \mathcal{F}}{\partial a} .
$$

This equation is to be interpreted as an equality of formal power series in $\epsilon_{1}$.

\subsubsection{Seiberg-Witten data and proof of the Matone relation}

To leading order in $\epsilon_{1}$, we find

$$
\lambda_{0}:=\sqrt{m^{2} \wp-u} d z, \quad u:=2 \pi i \partial_{\tau} \mathcal{F}_{0} .
$$

We first determine the Riemann surface on which the square root appearing in the differential is single-valued. The Weierstrass $\wp$-function provides a two-to-one mapping from the torus to the sphere. The equation $m^{2} \wp=u$ hence has two solutions on the torus. Singlevaluedness of the differential $\lambda_{0}$ requires a branchcut connecting them. The natural home of the differential is therefore a curve of genus two. The curve degenerates at $\frac{u}{m^{2}}=e_{i}$, with $e_{i}$ any of the half-periods of the domain torus of $\wp$. By writing

$$
t^{2}=m^{2} \wp-u, \quad y^{2}=4 \prod_{i=1}^{3}\left(\wp-e_{i}\right),
$$

we can present the genus two Riemann surface in its hyperelliptic form,

$$
y^{2}=4 \prod_{i}\left(\frac{t^{2}+u}{m^{2}}-e_{i}\right)
$$

with holomorphic one-forms $\omega_{i}$

$$
\omega_{1}=2 \frac{d t}{y}=\frac{m^{2} d z}{\sqrt{m^{2} \wp-u}}, \quad \omega_{2}=2 \frac{t d t}{y}=m^{2} d z
$$

We will denote the cycles on the genus two surface as $A_{ \pm}$and $B_{ \pm}$, with $A, B$ specifying the cycle on each sheet (which has the topology of a torus) and \pm specifying the sheet. Thus,

and in particular,

$$
\oint_{A_{+}, B_{+}} \omega_{i}=-\oint_{A_{-}, B_{-}} \omega_{i}
$$

$$
\oint_{A_{ \pm}} \lambda_{0}= \pm 2 \pi i a
$$

Defining the dual period

$$
2 \pi i a_{D}^{0}:=\oint_{B_{+}} \lambda_{0}
$$

we will prove that

$$
2 \pi i \frac{\partial a_{D}^{0}}{\partial \tau}=-\frac{1}{4 \pi i} \frac{\partial u}{\partial a}
$$

where the $a$-dependence of $u$ is determined by equation (3.18). Integrating this relation with regard to $\tau$, we will thus obtain the equality of the $B_{+}$period of the logarithm of the semi-classical two-point conformal block and the $a$-derivative of the logarithm of the one-point block, up to a $\tau$ independent function. 
The gauge theoretic point of view. From a gauge theory perspective, with the modulus $u$ given as in (3.13) and $\mathcal{F}_{0}$ identified as the prepotential of the gauge theory via the $2 \mathrm{~d} / 4 \mathrm{~d}$ correspondence, this equality demonstrates that the $a$-derivative of the prepotential is the $B_{+}$period of $\lambda_{0}$, thus justifying identifying $\lambda_{0}$ as the Seiberg-Witten differential on the Seiberg-Witten curve (3.15).

Note that in the original formalism of Seiberg and Witten, the curve of a rank one gauge theory has genus one. In [23], precisely the genus two curve (3.15) appears, with a prescription for recovering the Seiberg-Witten data from the higher dimensional Jacobian. The interpretation of the full Jacobian is as follows: the ratio of $B_{+}$to $A_{+}$period of $\omega_{2}$ yields the ultraviolet coupling $\tau$ of the theory, while the ratio of the corresponding periods of $\omega_{1}$ yields the infrared coupling as determined by $\lambda_{0}$ as Seiberg-Witten differential. Exchanging + for - cycles merely changes signs in accord with (3.17). In [24, 25], the Seiberg-Witten curves of $\mathrm{SU}(2)$ superconformal Seiberg-Witten theories are proposed to generally arise as the double cover of curves parametrized by the ultraviolet couplings of the theory.

The proof of equation (3.20) can also be interpreted from the conventional angle, in which $u$ is a coordinate on the gauge theory moduli space, a priori unrelated to the prepotential $\mathcal{F}_{0}$. The latter is introduced via its relation to the dual period of the SeibergWitten differential, $\partial \mathcal{F}_{0} / \partial a=-4 \pi i a_{D}^{0}$. The equation (3.20) then becomes the $a$-derivative of the Matone relation for $\mathcal{N}=2^{*}$ gauge theory (up to an $a$-independent term in $\mathcal{F}_{0}$, which carries no physical interpretation),

$$
2 \pi i \frac{\partial \mathcal{F}_{0}(a, \tau)}{\partial \tau}=u
$$

The proof that will follow hence also provides the first demonstration of this equation for $\mathcal{N}=2^{*}$ purely within the Seiberg-Witten framework. A demonstration using instanton calculus has appeared in [13].

The proof. For simplicity of notation, we will set $m^{2}=1$ in the following. The $m$ dependence can easily be restored via dimensional analysis, by assigning mass dimension 2 to $u$.

The proof we present is a variant of the proof of the Riemann bilinear identity. We define the function

$$
\eta_{0}(z)=\int^{z} \frac{1}{\sqrt{\wp-u}} d z^{\prime} .
$$

We will calculate the integral of

$$
\eta_{0}(z) \partial_{\tau} \lambda_{0}
$$

along the parallelogram in the complex plane spanned by 1 and $\tau$ in two ways: by integrating along the edges of the parallelogram, and alternatively, by contracting the contour inside the torus to hug the branch cut. For the first method, we will make use of the identities (see e.g. [26])

$$
\partial_{\tau} \wp(z+1, \tau)=\partial_{\tau \wp}(z, \tau), \quad \partial_{\tau} \wp(z+\tau, \tau)=\partial_{\tau} \wp(z, \tau)-\wp^{\prime}(z)
$$


We will denote the periods of the one-form $\omega_{1}$ along the cycles $A_{+}$and $B_{+}$as $\Pi_{A}, \Pi_{B}$ respectively. The evaluation on the parallelogram then proceeds as

$$
\begin{aligned}
2 \int_{0} \eta_{0}(z) \partial_{\tau} \lambda_{0}= & \int_{0}^{\tau} \frac{\left(\eta_{0} \partial_{\tau}(\wp-u)\right)(z+1)-\left(\eta_{0} \partial_{\tau}(\wp-u)\right)(z)}{\sqrt{\wp-u}} d z \\
& +\int_{0}^{1} \frac{\left(\eta_{0} \partial_{\tau}(\wp-u)\right)(z)-\left(\eta_{0} \partial_{\tau}(\wp-u)\right)(z+\tau)}{\sqrt{\wp-u}} d z \\
= & \int_{0}^{\tau} \frac{\left(\eta_{0}(z+1)-\eta_{0}(z)\right) \partial_{\tau}(\wp-u)(z)}{\sqrt{\wp-u}} d z \\
& +\int_{0}^{1} \frac{\eta_{0}(z) \partial_{\tau}(\wp-u)(z)-\left(\eta_{0}(z)+\Pi_{B}\right)\left(\partial_{\tau}(\wp-u)(z)-\wp^{\prime}(z)\right)}{\sqrt{\wp-u}} d z \\
= & \Pi_{A} \int_{0}^{\tau} \frac{\partial_{\tau}(\wp-u)(z)}{\sqrt{\wp-u}} d z+\int_{0}^{1} \frac{\left(\eta_{0} \wp^{\prime}\right)(z)}{\sqrt{\wp-u}} d z \\
& -\Pi_{B} \int_{0}^{1} \frac{\partial_{\tau}(\wp-u)(z)}{\sqrt{\wp-u}} d z+\Pi_{B} \int_{0}^{1} \frac{\wp^{\prime}(z)}{\sqrt{\wp-u}} d z
\end{aligned}
$$

The last two terms in equation (3.25) vanish. The first of these does so because $a$ is assumed to be $\tau$ independent. The second term in equation (3.25) can be further manipulated:

$$
\begin{aligned}
\int_{0}^{1} \frac{\eta_{0} \wp^{\prime}}{\sqrt{\wp-u}} d z & =2 \int_{0}^{1} \eta_{0} \frac{\partial}{\partial z} \sqrt{\wp-u} d z \\
& =2 \int_{0}^{1} \frac{\partial}{\partial z}\left(\eta_{0} \sqrt{\wp-u}\right) d z-2 \int_{0}^{1} \sqrt{\wp-u} \frac{\partial}{\partial z} \eta_{0} d z \\
& =\left.2 \eta_{0} \sqrt{\wp-u}\right|_{0} ^{1}-2 \\
& =2 \sqrt{\wp-u}(0)\left(\eta_{0}(1)-\eta_{0}(0)\right)-2 \\
& =2 \Pi_{A} \sqrt{\wp-u}(0)-2 .
\end{aligned}
$$

We hence find

$$
2 \int_{\square} \eta_{0}(z) \partial_{\tau} \lambda_{0}=2 \Pi_{A} \partial_{\tau} \int_{0}^{\tau} \sqrt{\wp-u} d z-2
$$

which contains the term we wish to evaluate.

Now, we compute the integral of the form (3.23) over the parallelgram again, this time by first contracting the integration contour to hug the branchcut between the two 
$\wp$-preimages of $u$ :

$$
\begin{aligned}
2 \int \eta_{0}(z) \partial_{\tau} \lambda_{0} d z & =\int \frac{\eta_{0}\left(z_{-}\right) \partial_{\tau}\left(\wp\left(z_{-}\right)-u\right)}{\sqrt{\wp\left(z_{-}\right)-u}} d z_{-}+\int \frac{\eta_{0}\left(z_{+}\right) \partial_{\tau}\left(\wp\left(z_{+}\right)-u\right)}{\sqrt{\wp\left(z_{+}\right)-u}} d z_{+} \\
& =\int \frac{\left(\eta_{0}\left(z_{-}\right)+\eta_{0}\left(z_{+}\right)\right) \partial_{\tau}\left(\wp\left(z_{-}\right)-u\right)}{\sqrt{\wp\left(z_{-}\right)-u}} d z_{-} \\
& =2 \eta_{0}\left(\wp^{-1}(u)_{1}\right) \int \frac{\partial_{\tau}\left(\wp\left(z_{-}\right)-u\right)}{\sqrt{\wp\left(z_{-}\right)-u}} d z_{-} \\
& =\eta_{0}\left(\wp^{-1}(u)_{1}\right) \int \frac{\partial_{\tau}(\wp(z)-u)}{\sqrt{\wp(z)-u}} d z \\
& =\eta_{0}\left(\wp^{-1}(u)_{1}\right) \int_{0}^{1} \frac{\wp^{\prime}}{\sqrt{\wp-u}} d z \\
& =\left.\eta_{0}\left(\wp^{-1}(u)_{1}\right) 2 \sqrt{\wp-u}\right|_{0} ^{1} \\
& =0
\end{aligned}
$$

where $\wp^{-1}(u)_{1}$ denotes the $\wp$-preimage of $u$ at the lower end of the branch cut (with regard to the diagram). We have thus arrived at the equality

$$
\Pi_{A} \partial_{\tau} \oint_{B_{+}} \lambda_{0}=1 \quad \Leftrightarrow \quad 2 \pi i \partial_{\tau} a_{D}=-\frac{1}{2} \frac{\partial \partial_{\tau} \mathcal{F}_{0}}{\partial a} \quad \Leftrightarrow \quad 2 \pi i a_{D}=-\frac{1}{2} \frac{\partial \mathcal{F}_{0}}{\partial a}+g(a),
$$

with $g(a)$ independent of $\tau$. Note that the differential equation (3.6) and its boundary condition (3.7) determine $\mathcal{F}$ only up to a $\tau$ independent piece. We are hence free to define $\mathcal{F}$ and in particular $\mathcal{F}_{0}$ such that $g(a)=0$, and hence

$$
\frac{\partial \mathcal{F}_{0}}{\partial a}=-2 \oint_{B_{+}} \lambda_{0}
$$

We will return to this point below after extending this equality beyond leading order in $\epsilon_{1}$, and determine the necessary integration constant that must be included in $\mathcal{F}$ for the equality (3.30) to hold.

Strictly speaking, to avoid integrating over the pole of the function $\wp$, we should shift all contours in our proof by a constant amount. This will eliminate the infinities otherwise present in intermediate steps in the calculation.

\subsubsection{Beyond leading order: quantum geometry}

Our results from [7] yield $\mathcal{W}^{\prime \prime}$ as a formal power series

$$
\frac{1}{a} \mathcal{W}^{\prime \prime} \in \mathbb{C}\left[E_{2}, E_{4}, E_{6}, \wp, \wp^{\prime}\right]\left[\left[\frac{m}{a}\right]\right]\left[\left[\frac{\epsilon_{1}}{a}\right]\right] .
$$


The coefficients of the formal series in $\frac{\epsilon_{1}}{a}$ are convergent power series in $\frac{m}{a}$. Interpreted as equalities between formal power series in $\frac{\epsilon_{1}}{a}$, the above calculation goes through almost unchanged, with the differential $\lambda_{0}$ replaced by the differential $\lambda$, the function $\eta_{0}$ replaced by

$$
\eta(z)=\int^{z} \frac{d z}{\sqrt{\left(1-\frac{\epsilon_{1}^{2}}{4}\right) \wp-U-\epsilon_{1} \mathcal{W}^{\prime \prime}}}, \quad U=2 \pi i \partial_{\tau} \mathcal{F},
$$

and the periods $\Pi_{A}, \Pi_{B}$ defined as the $A_{+}$and $B_{+}$periods of

$$
\Omega_{1}=\frac{d z}{\sqrt{\left(1-\frac{\epsilon_{1}^{2}}{4}\right) \wp-U-\epsilon_{1} \mathcal{W}^{\prime \prime}}} .
$$

The argument now relies, in addition to the quasi-periodicity properties (3.24) of the derivative of the Weierstrass function $\partial_{\tau} \wp$, on the equality

$$
\begin{aligned}
\partial_{\tau} \mathcal{W}^{\prime \prime}(z+\tau) & =\partial_{\tau}^{E} \mathcal{W}^{\prime \prime}(z+\tau)+\partial_{\wp} \mathcal{W}^{\prime \prime}(z+\tau) \partial_{\tau \wp}(z+\tau)+\partial_{\wp} \mathcal{W}^{\prime \prime}(z+\tau) \partial_{\tau} \wp^{\prime}(z+\tau) \\
& =\partial_{\tau} \mathcal{W}^{\prime \prime}(z)-\partial_{\wp} \mathcal{W}^{\prime \prime}(z) \wp^{\prime}(z)-\partial_{\wp^{\prime}} \mathcal{W}^{\prime \prime}(z) \wp^{\prime \prime}(z) \\
& =\partial_{\tau} \mathcal{W}^{\prime \prime}(z)-\mathcal{W}^{\prime \prime \prime}(z)
\end{aligned}
$$

The notation $\partial_{\tau}^{E} \mathcal{W}^{\prime \prime}$ is used to indicate the derivative of $\mathcal{W}^{\prime \prime}$ with regard to the $\tau$-dependence of the quasi-modular forms in the presentation (3.31) of $\mathcal{W}^{\prime \prime}$. We can thus establish the equality between the $a$-derivative of $\mathcal{F}$ and a period integral over a formal power series involving the $\tau$-derivative of $\mathcal{F}$,

$$
\frac{\partial \mathcal{F}}{\partial a}=-2 \oint_{B_{+}} \sqrt{\wp-2 \pi i \partial_{\tau} \mathcal{F}-\epsilon_{1} \mathcal{W}^{\prime \prime}-\epsilon_{1}^{2} \frac{\wp}{4}} d z+G(a),
$$

with $G(a)$ a function independent of $\tau$. As above, we wish to define $\mathcal{F}$ to incorporate $G(a)$. To specify the ensuing integration constant in passing from $\partial_{\tau} \mathcal{F}$ to $\mathcal{F}$, note that (3.35) can be seen as an infinite set of equalities between polynomials in the three independent variables $E_{2}, E_{4}, E_{6}$. Setting these to zero, the integral on the right hand side can be evaluated in a large $a$ expansion to give

$$
\begin{aligned}
\left.\oint_{B_{+}} \sqrt{\wp-2 \pi i \partial_{\tau} \mathcal{F}-\epsilon_{1} \mathcal{W}^{\prime \prime}-\epsilon_{1}^{2} \frac{\wp}{4}} d z\right|_{E_{i}=0} & =\left.\oint_{B_{+}} \sqrt{\left(1-\frac{\epsilon_{1}^{2}}{4}\right) \wp+(2 \pi i a)^{2}} d z\right|_{E_{i}=0} \\
& =2 \pi i a\left(\tau+\frac{1}{2} \frac{1}{(2 \pi i a)^{2}}\left(1-\frac{\epsilon_{1}^{2}}{4}\right) 2 \pi i\right) \\
& =2 \pi i a \tau+\frac{1}{2 a}\left(1-\frac{\epsilon_{1}^{2}}{4}\right) .
\end{aligned}
$$

We have used that $\left.\mathcal{W}^{\prime \prime}\right|_{E_{i}=0}$ is a formal power series with coefficients in $\wp^{2} \mathbb{C}[\wp] \oplus \wp^{\prime} \mathbb{C}[\wp]$, that $\left.\oint_{B} \wp d z\right|_{E_{i}=0}=2 \pi i,\left.\oint_{B} \wp^{n} d z\right|_{E_{i}=0}=0$ for $n>1[27]$, and that $\left.\partial_{\tau} \mathcal{F}\right|_{E_{i}=0}=-2 \pi i a^{2}$. If we hence choose the integration constant in passing from $\partial_{\tau} \mathcal{F}$ to $\mathcal{F}$ such that

$$
\left.\frac{\partial \mathcal{F}}{\partial a}\right|_{E_{i}=0}=-4 \pi i a \tau-\frac{1}{a}\left(1-\frac{\epsilon_{1}^{2}}{4}\right)
$$


we set the $\tau$ independent function $G(a)$ in (3.35) to zero, and obtain the relation between the $\epsilon_{1}$-deformed prepotential and Seiberg-Witten differential in the final form

$$
\frac{\partial \mathcal{F}}{\partial a}=-2 \oint_{B_{+}} \sqrt{\wp-2 \pi i \partial_{\tau} \mathcal{F}-\epsilon_{1} \mathcal{W}^{\prime \prime}-\epsilon_{1}^{2} \frac{\wp}{4}} d z
$$

The equality (3.38) permits us to fill a gap in our results in [7]. There, we demonstrated that $\partial_{\tau} \mathcal{F}_{n}$ are quasi-modular, and we verified experimentally at low $n$ that this property is inherited from $\mathcal{F}_{n}$. As derivatives with respect to the variable $a$ do not interfere with quasimodularity and the right hand side of (3.38) is manifestly quasi-modular, the relation (3.38) proves the quasi-modularity of $\mathcal{F}_{n}$ to all orders.

Note that if the functions $\mathcal{F}$ and $\mathcal{W}$ were analytic, rather than formal power series, the right hand side of equation (3.35) could be interpreted as the integral of a meromorphic form over a modified (or quantum-corrected) Seiberg-Witten geometry, which would depend on the solutions to the equation $\wp=U+\epsilon_{1} \mathcal{W}^{\prime \prime}+\epsilon_{1}^{2} \frac{\wp}{4}$.

A final remark on the null vector decoupling equation (3.2) is that we can think of the differential equation as the quantization of a deformed Seiberg-Witten curve with the operator $\partial_{z}$ and the variable $z$ as canonically conjugate variables. The null vector decoupling equation on the torus can thus be interpreted as the quantum curve annihilating the partition function.

Several other approaches to deformed Seiberg-Witten theory have appeared in the literature. In the topological string setting, the notions of deformed periods and curves elevated to differential operators annihilating the partition function were introduced and studied in [28-31]. For results inspired by the relation between the $\epsilon_{2} \rightarrow 0$ limit and integrable models, see [32, 33]. A matrix model approach is developed in [18, 34-37].

\subsubsection{Comparing to the proposal in [1] for the Seiberg-Witten geometry}

Above, we have demonstrated that the differential $\mathcal{W}^{\prime} d z$, defined in the semi-classical $\epsilon_{2} \rightarrow 0$ limit via equation (3.4), can be identified with the Seiberg-Witten differential. Defining the Seiberg-Witten curve by the requirement that this one-form be single-valued, we obtained the hyperelliptic equation for the curve to be

$$
t^{2}=\left(\mathcal{W}^{\prime}\right)^{2}=\left(\left.\lim _{\epsilon_{2} \rightarrow 0} \frac{d}{d z} \log \left\langle V_{h_{m}}(0) V_{h_{(2,1)}}(z)\right\rangle_{\tau}\right|_{h_{, h_{ \pm}}}\right)^{2}
$$

The choice of the second internal momentum $h_{ \pm}$simply determines the overall sign of $\mathcal{W}^{\prime}$. The following Seiberg-Witten curve was proposed in $[1]:^{1}$

$$
t^{2}=\lim _{\epsilon_{2} \rightarrow 0} \epsilon_{1} \epsilon_{2} \frac{\left.\left\langle T(z) V_{h_{m}}(0)\right\rangle_{\tau}\right|_{h}}{\left.\left\langle V_{h_{m}}(0)\right\rangle_{\tau}\right|_{h}}
$$

\footnotetext{
${ }^{1}$ We have adjusted the limit to our parametrization of the variables.
} 
with Seiberg-Witten differential $t d z$. To compare the two proposals, we can evaluate the right hand side of equation (3.40) by invoking the Ward identity [38]:

$$
\begin{aligned}
& \left\langle T(z) \prod_{i=1}^{n} V_{i}\left(z_{i}\right)\right\rangle-\langle T\rangle\left\langle\prod_{i=1}^{n} V_{i}\left(z_{i}\right)\right\rangle= \\
& \sum_{i=1}^{n}\left(h_{i}\left(\wp\left(z-z_{i}\right)+2 \eta_{1}\right)+\left(\zeta\left(z-z_{i}\right)+2 \eta_{1} z_{i}\right) \partial_{z_{i}}\right)\left\langle\prod_{i=1}^{n} V_{i}\left(z_{i}\right)\right\rangle+2 \pi i \partial_{\tau}\left\langle\prod_{i=1}^{n} V_{i}\left(z_{i}\right)\right\rangle .
\end{aligned}
$$

We obtain

$$
\frac{\left\langle T(z) V_{h_{m}}(0)\right\rangle}{\left\langle V_{h_{m}}(0)\right\rangle}=h_{m}\left(\wp(z)+2 \eta_{1}\right)+2 \pi i \partial_{\tau} \log \langle V(0)\rangle+\langle T\rangle .
$$

Projecting this equation onto the $h$ channel and substituting our definition of the one-point conformal block $\mathcal{F}$ from equation (3.5) yields

$$
\lim _{\epsilon_{2} \rightarrow 0} \epsilon_{1} \epsilon_{2} \frac{\left.\left\langle T(z) V_{h_{m}}(0)\right\rangle\right|_{h}}{\left.\left\langle V_{h_{m}}(0)\right\rangle\right|_{h}}=\left(\frac{\epsilon_{1}^{2}}{4}-m^{2}\right) \wp(z)+2 \pi i \partial_{\tau} \mathcal{F},
$$

where we have used $\langle T\rangle=2 \pi i \partial_{\tau} \log Z$. This is to be compared to the expression (3.6) for $\left(\mathcal{W}^{\prime}\right)^{2}$ we obtain by imposing null vector decoupling,

$$
\epsilon_{1}^{2}\left\langle\left(L_{-2} V_{(2,1)}\right)(w) V_{h_{m}}(0)\right\rangle=\frac{1}{\epsilon_{2}^{2}}\left\langle\left(L_{-1}^{2} V_{(2,1)}\right)(w) V_{h_{m}}(0)\right\rangle .
$$

Projecting onto the $h, h_{ \pm}$channel, dividing both sides of this equation by the two-point block, and taking the $\epsilon_{2} \rightarrow 0$ limit yields [7]

$$
\left(\mathcal{W}^{\prime}\right)^{2}+\epsilon_{1} \mathcal{W}^{\prime \prime}=\left(\frac{\epsilon_{1}^{2}}{4}-m^{2}\right) \wp(z)+2 \pi i \partial_{\tau} \mathcal{F} .
$$

The proposals in equations (3.39) and (3.40) hence yield the same classical Seiberg-Witten curve (defined at $\epsilon_{1}=0$ ). The additional term $\epsilon_{1} \mathcal{W}^{\prime \prime}$ arising from (3.39) enters into the definition of the deformed Seiberg-Witten differential (3.11) and is necessary for reproducing the amplitudes $\mathcal{F}_{n}$ expected from gauge theory beyond the lowest order in $\epsilon_{1}$.

\subsubsection{Bohr-Sommerfeld interpretation}

Our analysis can be cast in the light of a Bohr-Sommerfeld evaluation of the Schrödingerlike equation (3.2) in the $\epsilon_{2} \rightarrow 0$ limit,

$$
\left(-\epsilon_{1}^{2} \partial_{z}^{2}+V(z)\right) \Psi(z \mid \tau)=u \Psi(z \mid \tau)
$$

with

$$
V(z)=\left(m^{2}-\frac{\epsilon_{1}^{2}}{4}\right) \wp(z), \quad u=2 \pi i \partial_{\tau} \mathcal{F} .
$$

It was pointed out in $[17,18]$ that the $2 \mathrm{~d} / 4 \mathrm{~d}$ correspondence permits determining Schrödinger equations associated to a Seiberg-Witten theory via null vector decoupling equations. The analysis closest in spirit to ours appears in [34], where the sine-Gordon quantum model is used to compute the $\epsilon_{1}$-deformed prepotential of pure $\mathrm{SU}(2)$ gauge theory. The 
authors compute the $A$ and $B$ periods $\Pi_{A}$ and $\Pi_{B}$ of the exact Bohr-Sommerfeld integral ( $\lambda$ in the notation above) as functions of $u$, invert $a=\Pi_{A}(u)$ to obtain $u(a)$, and then impose $\frac{\partial F\left(a \mid \epsilon_{1}\right)}{\partial a}=\Pi_{B}(u(a))$ to determine $F\left(a \mid \epsilon_{1}\right)$. They establish to low orders in $\epsilon_{1}$ that the $F\left(a \mid \epsilon_{1}\right)$ thus computed coincides with the $\epsilon_{1}$-deformed prepotential, thereby providing evidence for a claim in [11]. Given the $2 \mathrm{~d} / 4 \mathrm{~d}$ correspondence, our analysis above in fact proves to all orders that this procedure must yield the deformed prepotential: the $2 \mathrm{~d} / 4 \mathrm{~d}$ correspondence identifies the $\mathcal{F}$ appearing in (3.47) with this prepotential, while we proved in subsection 3.2.3 that the $B$ period of $\lambda$ coincides with the $a$-derivative of $\mathcal{F}$.

\subsection{Transformation properties and S-duality}

The quasi-modular $\tau$-dependence of the coefficients of $\mathcal{F}$ in a formal $\epsilon_{1}$-expansion is clearly a consequence of $S$-duality in gauge theory, through the $2 \mathrm{~d} / 4 \mathrm{~d}$ correspondence. Encountering quasi-modularity in this context may be surprising at first blush, because such forms in fact transform in a rather messy way under the $S$-transformation of the modular group $\mathrm{SL}(2, \mathbb{Z})$.

Electromagnetic duality states that the same $\mathcal{N}=2$ gauge theory expressed in terms of electric or magnetic variables has infrared couplings related by $\tau_{D}^{\mathrm{IR}}=-1 / \tau^{\mathrm{IR}}$. The derivatives of the corresponding prepotentials, $h=F^{\prime}(a)$ and $h_{D}=F^{\prime}\left(a_{D}\right)$, must hence be inverse functions of each other, up to a sign [9]: $h_{D}(h(a))=-a$. Two functions whose derivatives are inverse functions of each other are themselves related by Legendre transform, hence $F_{D}\left(a_{D}\right)=F(a)-a_{D} a$.

S-duality identifies superconformal theories specified by different ultraviolet data. In the case of $\mathcal{N}=2^{*}$, we will demonstrate, using our conformal field theory approach, that the prepotentials of the theories at ultraviolet couplings $\tau$ and $-1 / \tau$ behave as $F$ to $F_{D}$, in the sense that

$$
F\left(a_{D} ;-1 / \tau\right)=F(a ; \tau)-a_{D} a
$$

We will furthermore prove that this relation persists to all orders in $\epsilon_{1}$. To this end, we introduce the notation $\mathcal{F}(\tau ; a)$ and $\mathcal{W}(z, \tau ; a)$ to indicate the unique solution of the differential equation (3.6) satisfying the boundary condition

$$
\oint_{A_{+}} \mathcal{W}^{\prime}(z, \tau ; a)=2 \pi i a
$$

and

$$
\oint_{A_{+}} \sqrt{\left(1-\frac{\epsilon_{1}^{2}}{4}\right) \wp(z, \tau)-2 \pi i \partial_{\tau} \mathcal{F}(\tau ; a)-\epsilon_{1} \partial_{z}^{2} \mathcal{W}(z, \tau ; a)} d z=2 \pi i a
$$


Note that as long at the $\partial_{\tau}$ derivative on $\mathcal{F}$ is defined as acting only on the first argument, we are also entitled to endow $a$ with $\tau$-dependence. Let us now consider

$$
\begin{aligned}
2 \pi i a_{D}(-1 / \tau) & =\oint_{B_{+}} \sqrt{\left(1-\frac{\epsilon_{1}^{2}}{4}\right) \wp\left(z,-\frac{1}{\tau}\right)-2 \pi i \partial_{1} \mathcal{F}\left(-\frac{1}{\tau} ; a\right)-\epsilon_{1} \partial_{z}^{2} \mathcal{W}\left(z,-\frac{1}{\tau} ; a\right)} d z \\
& =\int_{0}^{-\frac{1}{\tau}} \sqrt{\left(1-\frac{\epsilon_{1}^{2}}{4}\right) \wp\left(z,-\frac{1}{\tau}\right)-2 \pi i \partial_{1} \mathcal{F}\left(-\frac{1}{\tau} ; a\right)-\epsilon_{1} \partial_{z}^{2} \mathcal{W}\left(z,-\frac{1}{\tau} ; a\right)} d z \\
& =\frac{1}{\tau} \int_{0}^{-1} \sqrt{\left(1-\frac{\epsilon_{1}^{2}}{4}\right) \wp\left(\frac{z}{\tau},-\frac{1}{\tau}\right)-2 \pi i \partial_{1} \mathcal{F}\left(-\frac{1}{\tau} ; a\right)-\epsilon_{1} \partial_{1}^{2} \mathcal{W}\left(\frac{z}{\tau},-\frac{1}{\tau} ; a\right)} d z \\
& =-\int_{0}^{1} \sqrt{\left(1-\frac{\epsilon_{1}^{2}}{4}\right) \wp(z, \tau)-2 \pi i \partial_{\tau} \mathcal{F}\left(-\frac{1}{\tau} ; a\right)-\epsilon_{1} \partial_{z}^{2} \mathcal{W}\left(\frac{z}{\tau},-\frac{1}{\tau} ; a\right)} d z .
\end{aligned}
$$

In the last line, we have used the fact that both $\wp$ and $\mathcal{W}^{\prime \prime}(z / \tau,-1 / \tau)$ are invariant under $z \rightarrow z+1$, the latter via (3.31). By considering the asymptotic expansion, and the lowest order in $\epsilon_{1}$ explicitly, we conclude that

$2 \pi i \partial_{\tau} \mathcal{F}\left(-\frac{1}{\tau} ; a\right)+\epsilon_{1} \partial_{z}^{2} \mathcal{W}\left(\frac{z}{\tau},-\frac{1}{\tau} ; a\right)=2 \pi i \partial_{1} \mathcal{F}\left(\tau ;-a_{D}(-1 / \tau)\right)+\epsilon_{1} \partial_{z}^{2} \mathcal{W}\left(z, \tau ;-a_{D}(-1 / \tau)\right)$

Calculating the $A_{+}$period of both sides and invoking (3.31) finally yields

$$
\partial_{\tau} \mathcal{F}\left(-\frac{1}{\tau} ; a\right)=\partial_{1} \mathcal{F}\left(\tau ; a_{D}(-1 / \tau)\right)
$$

or equivalently

$$
\partial_{\tau} \mathcal{F}(\tau ; a)=\partial_{\tau} \mathcal{F}\left(-1 / \tau ; a_{D}(\tau)\right)
$$

with the $\tau$-derivative on the right hand side only acting on the first argument of $\mathcal{F}$. We have here used the fact that $\mathcal{F}$ is an even function of $a$. Since $\partial_{z} \mathcal{W}$ is odd under $(z, a) \rightarrow$ $-(z, a)[7]$, this allows us to conclude that

$$
\partial_{z}^{2} \mathcal{W}(z, \tau ; a)=\partial_{z}^{2} \mathcal{W}\left(\frac{z}{\tau},-\frac{1}{\tau} ; a_{D}(\tau)\right)
$$

To integrate (3.54), we need to pass from partial to total $\tau$-derivatives. Assuming that $a$ is $\tau$ independent, this is

$$
\frac{d}{d \tau} \mathcal{F}(\tau ; a)=\frac{d}{d \tau} \mathcal{F}\left(-1 / \tau ; a_{D}\right)-\partial_{2} \mathcal{F}\left(-1 / \tau ; a_{D}\right) \frac{d a_{D}}{d \tau}
$$


Starting from (3.38), a calculation very similar to (3.51) invoking (3.54) and (3.55) yields

$$
\begin{aligned}
\partial_{2} \mathcal{F} & \left.-1 / \tau ; a_{D}\right) \\
& =-2 \oint_{B_{+}} \sqrt{\left(1-\frac{\epsilon_{1}^{2}}{4}\right) \wp\left(z,-\frac{1}{\tau}\right)-2 \pi i \partial_{1} \mathcal{F}\left(-\frac{1}{\tau} ; a_{D}\right)-\epsilon_{1} \partial_{1}^{2} \mathcal{W}\left(z,-\frac{1}{\tau} ; a_{D}\right)} d z \\
& =-2 \int_{0}^{-\frac{1}{\tau}} \sqrt{\left(1-\frac{\epsilon_{1}^{2}}{4}\right) \wp\left(z,-\frac{1}{\tau}\right)-2 \pi i \partial_{1} \mathcal{F}\left(-\frac{1}{\tau} ; a_{D}\right)-\epsilon_{1} \partial_{1}^{2} \mathcal{W}\left(z,-\frac{1}{\tau} ; a_{D}\right)} d z \\
& =-\frac{2}{\tau} \int_{0}^{-1} \sqrt{\left(1-\frac{\epsilon_{1}^{2}}{4}\right) \wp\left(\frac{z}{\tau},-\frac{1}{\tau}\right)-2 \pi i \partial_{1} \mathcal{F}\left(-\frac{1}{\tau} ; a_{D}\right)-\epsilon_{1} \partial_{1}^{2} \mathcal{W}\left(\frac{z}{\tau},-\frac{1}{\tau} ; a_{D}\right)} d z \\
& =2 \int_{0}^{1} \sqrt{\left(1-\frac{\epsilon_{1}^{2}}{4}\right) \wp(z, \tau)-2 \pi i \partial_{\tau} \mathcal{F}\left(-\frac{1}{\tau} ; a_{D}\right)-\epsilon_{1} \partial_{z}^{2} \mathcal{W}\left(\frac{z}{\tau},-\frac{1}{\tau} ; a_{D}\right)} d z \\
& =2 \int_{0}^{1} \sqrt{\left(1-\frac{\epsilon_{1}^{2}}{4}\right) \wp(z, \tau)-2 \pi i \partial_{\tau} \mathcal{F}(\tau ; a)-\epsilon_{1} \partial_{z}^{2} \mathcal{W}(z, \tau ; a)} d z \\
& =4 \pi i a .
\end{aligned}
$$

Hence,

$$
\mathcal{F}\left(-1 / \tau ; a_{D}\right)=\mathcal{F}(\tau, a)+4 \pi i a a_{D}+C .
$$

Taking the total $a$-derivative on both sides demonstrates that the constant $C$ is independent of $a$ as well as of $\tau$. Explicit computation shows that the contributions to the constant stem from orders 0 and 1 in $\epsilon_{1}^{2}$, and that $C=-\frac{1}{2} \pi i\left(1-\frac{\epsilon_{1}^{2}}{4}\right)$.

The transformation properties of $\mathcal{F}$ under S-duality were studied in [39, 40] using matrix model techniques, and in [21] based on the holomorphic anomaly equations.

\section{Exact conformal field theory methods}

In the previous section, we computed the monodromy of the two-point conformal block (2.4) around the $B$-period of the torus and the transformation properties of the one-point conformal block (2.1) under the S-transformation $\tau \rightarrow-1 / \tau$ by analyzing the null vector decoupling equation in the semi-classical limit. Both computations can be performed exactly in conformal field theory. By re-deriving our results from the semi-classics of these exact relations, we demonstrate that it is consistent to take the semi-classical approximation already at the level of the null vector decoupling equations. Extracting the gauge theory/topological string theory amplitudes from these exact results is a first step towards moving beyond perturbation theory on this side of the $2 \mathrm{~d} / 4 \mathrm{~d}$ correspondence.

\subsection{The $B$-monodromy from braiding}

We can compute the $A$-monodromy of the two-point toroidal block in the position of the degenerate operator from the operator product expansions

$$
\begin{aligned}
& \phi_{h_{(2,1)}}(z)|a\rangle \\
& \quad=\phi_{h_{(2,1)}}(z) \phi_{a}(0)|0\rangle \\
& \quad=\left(C_{h_{(2,1)}, h_{a}}^{h_{+}} z^{h_{+}-h_{(2,1)}-h_{a}}\left(\phi_{h_{+}}(0)+\ldots\right)+C_{h_{(2,1)}, h_{a}}^{h_{-}} z^{h_{-}-h_{(2,1)}-h_{a}}\left(\phi_{h_{-}}(0)+\ldots\right)\right)|0\rangle,
\end{aligned}
$$




$$
\begin{aligned}
& \langle a| \phi_{h_{(2,1)}}(z)=\lim _{w \rightarrow \infty} w^{2 h_{a}}\langle 0| \phi_{h_{a}}(w) \phi_{h_{(2,1)}}(z) \\
& =\lim _{\tilde{w} \rightarrow 0} \tilde{z}^{2 h_{(2,1)}}\langle 0| \tilde{\phi}_{h_{a}}(\tilde{w}) \tilde{\phi}_{h_{(2,1)}}(\tilde{z}) \\
& =\langle 0|\left(C_{h_{a}, h_{(2,1)}}^{h_{+}}(-\tilde{z})^{h_{+}+h_{(2,1)}-h_{a}}\left(\phi_{h_{+}}(0)+\ldots\right)\right. \\
& \left.+C_{h_{a}, h_{(2,1)}}^{h_{-}}(-\tilde{z})^{h_{-}+h_{(2,1)}-h_{a}}\left(\phi_{h_{-}}(0)+\ldots\right)\right),
\end{aligned}
$$

by circling the origin or infinity respectively, obtaining the same two monodromies in the semi-classical limit. This avenue of computation is available as the operator product expansion remains valid along the entire path associated to the monodromy. By contrast, the $B$-monodromy requires exchanging the order of the two operator insertions along the monodromy path. Its computation hence requires invoking braiding matrices. These relate the conformal blocks

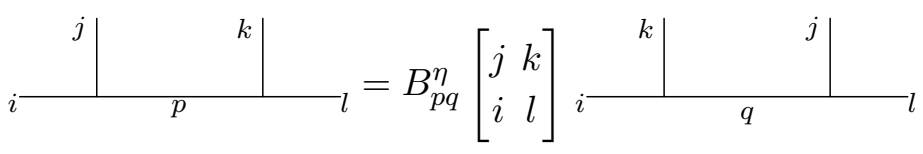

The index $\eta= \pm$ indicates the sense of the braiding. It will not play a role in the following. As usual, we glue the two ends of the diagram to obtain a torus conformal block by inserting a translation operator $q^{H}$ and summing over initial and final states. By choosing these states in an eigenbasis of $H$, we see that the braiding matrix is not affected by the insertion of this operator.

We can relate the two-point function evaluated at arguments $z$ and $z+\tau$ via the following sequence of manipulations:

$$
\begin{aligned}
Z\left(a, a_{+} ; z-w\right) & =\left\langle a\left|q^{H} \Phi_{h_{m}}(w)\right| a_{+}\right\rangle\left\langle a_{+}\left|\Phi_{h_{(2,1)}}(z)\right| a\right\rangle \\
& =\left\langle a_{+}\left|\Phi_{h_{(2,1)}}(z)\right| a\right\rangle\left\langle a\left|q^{H} \Phi_{h_{m}}(w)\right| a_{+}\right\rangle \\
& =\left\langle a_{+}\left|\Phi_{h_{(2,1)}}(z) q^{H}\right| a\right\rangle\left\langle a\left|\Phi_{h_{m}}(w)\right| a_{+}\right\rangle \\
& =\left\langle a_{+}\left|q^{H} \Phi_{h_{(2,1)}}(z+\tau)\right| a\right\rangle\left\langle a\left|\Phi_{h_{m}}(w)\right| a_{+}\right\rangle \\
& =\sum_{a^{\prime}=a, a_{++}} B_{a a^{\prime}}^{\eta}\left[\begin{array}{rr}
-b / 2 & \alpha_{m} \\
\alpha_{+} & \alpha_{+}
\end{array}\right]\left\langle a_{+}\left|q^{H} \Phi_{h_{m}}(w)\right| a^{\prime}\right\rangle\left\langle a^{\prime}\left|\Phi_{h_{(2,1)}}(z+\tau)\right| a_{+}\right\rangle
\end{aligned}
$$

with $a_{+}=a+\frac{\epsilon_{2}}{2}, a_{++}=a+\epsilon_{2}, \alpha_{+}=\frac{Q}{2}+\frac{a_{+}}{\sqrt{\epsilon_{1} \epsilon_{2}}}, \alpha_{m}=\frac{Q}{2}+\frac{m}{\sqrt{\epsilon_{1} \epsilon_{2}}}$. The notation here is that repeated states imply a sum over the descendants of the indicated primaries. 
Diagrammatically,
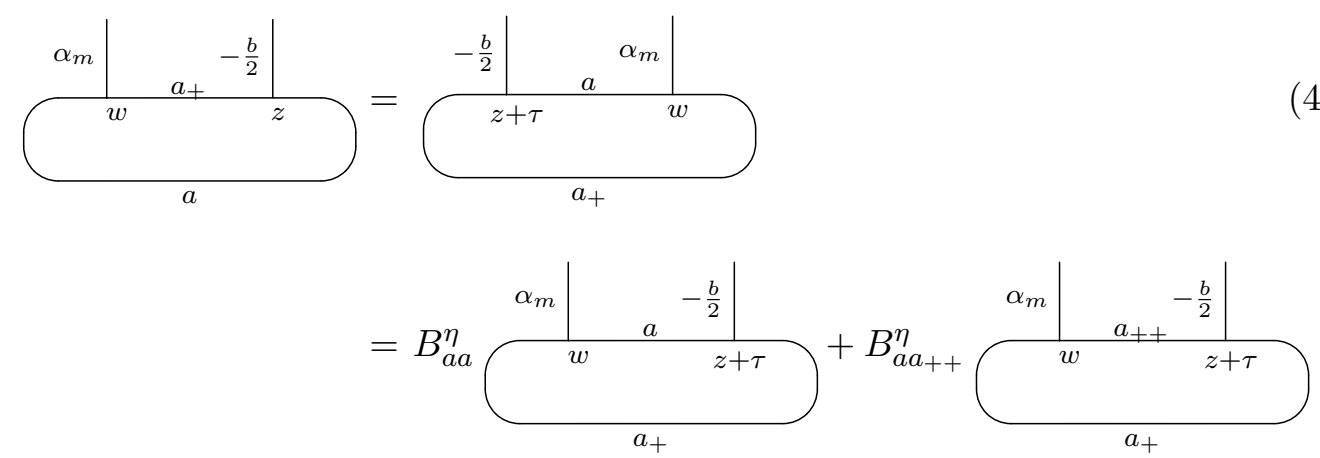

To justify the manipulations, we assume that an orthonormal basis for each level has been introduced. We can obtain braiding from fusion matrices via [41]

$$
F_{a a^{\prime}}\left[\begin{array}{ll}
\alpha_{2} & \alpha_{3} \\
\alpha_{1} & \alpha_{4}
\end{array}\right]=e^{-i \phi(\eta)} B_{a a^{\prime}}^{\eta}\left[\begin{array}{ll}
\alpha_{2} & \alpha_{4} \\
\alpha_{1} & \alpha_{3}
\end{array}\right]
$$

with $\phi(\eta)=\eta \pi\left(\Delta_{\alpha_{1}}+\Delta_{\alpha_{3}}-\Delta_{a}-\Delta_{a^{\prime}}\right)$. With the fusion matrices as derived in [14], we arrive at

$$
\begin{aligned}
& B_{a_{1-} a_{1-}}^{\eta}\left[\begin{array}{cr}
-b / 2 & \alpha_{2} \\
\alpha_{1} & \alpha_{1}
\end{array}\right]=e^{i \phi(\eta)} \frac{\Gamma\left[\left(2 \alpha_{1}-b\right) b\right] \Gamma\left[\left(Q-2 \alpha_{1}\right) b\right]}{\Gamma\left[\left(\alpha_{2}-\frac{b}{2}\right) b\right] \Gamma\left[1-\alpha_{2} b+\frac{b^{2}}{2}\right]} \\
& B_{a_{1-} a_{1+}}^{\eta}\left[\begin{array}{cr}
-b / 2 & \alpha_{2} \\
\alpha_{1} & \alpha_{1}
\end{array}\right]=e^{i \phi(\eta)} \frac{\Gamma\left[\left(2 \alpha_{1}-b\right) b\right] \Gamma\left[-\left(Q-2 \alpha_{1}\right) b\right]}{\Gamma\left[\left(2 \alpha_{1}-\alpha_{2}-\frac{b}{2}\right) b\right] \Gamma\left[\left(2 \alpha_{1}+\alpha_{2}-\frac{b}{2}-Q\right) b\right]},
\end{aligned}
$$

with $\alpha_{i}=\frac{Q}{2}+\frac{a_{i}}{\sqrt{\epsilon_{1} \epsilon_{2}}}$. Setting $\alpha_{1}=\alpha_{+}, \alpha_{2}=\alpha_{m}$, this yields

$$
\begin{aligned}
B_{a a}^{\eta} & =e^{i \phi_{1}(\eta)} \frac{\Gamma\left[-\frac{2 a+\epsilon_{2}}{\epsilon_{1}}\right] \Gamma\left[1+\frac{2 a+\epsilon_{2}}{\epsilon_{1}}\right]}{\Gamma\left[\frac{1}{2}-\frac{m}{\epsilon_{1}}\right] \Gamma\left[\frac{1}{2}+\frac{m}{\epsilon_{1}}\right]}, \\
B_{a a_{++}}^{\eta} & =e^{i \phi_{2}(\eta)} \frac{\Gamma\left[\frac{2 a+\epsilon_{2}}{\epsilon_{1}}\right] \Gamma\left[1+\frac{2 a+\epsilon_{2}}{\epsilon_{1}}\right]}{\Gamma\left[\frac{1}{2}+\frac{2 a-m+\epsilon_{2}}{\epsilon_{1}}\right] \Gamma\left[\frac{1}{2}+\frac{2 a+m+\epsilon_{2}}{\epsilon_{1}}\right]},
\end{aligned}
$$

where $\phi_{1}=-\eta \pi \frac{4 a+\epsilon_{2}}{2 \epsilon_{1}}$ and $\phi_{2}=\eta \pi \frac{\epsilon_{2}}{2 \epsilon_{1}}$. Using

$$
\begin{aligned}
\Gamma\left(\frac{1}{2}+x\right) \Gamma\left(\frac{1}{2}-x\right) & =\frac{\pi}{\cos \pi x}, \\
\Gamma(1+i x) \Gamma(1-i x) & =\frac{\pi x}{\sinh \pi x}, \quad x \in \mathbb{R},
\end{aligned}
$$

and

$$
\lim _{|z| \rightarrow \infty} \frac{\Gamma(z+a)}{\Gamma(z)} e^{-a \log z}=1,
$$

and setting $a=i \alpha, m=i \mu, \alpha, \mu \in \mathbb{R}$, we obtain

$$
B_{a a}^{\eta} \underset{\epsilon_{2} \rightarrow 0}{\longrightarrow}-i e^{-\eta i \pi \frac{2 \alpha}{\epsilon_{1}}} \frac{\cosh \pi \frac{\mu}{\epsilon_{1}}}{\sinh \pi \frac{2 \alpha}{\epsilon_{1}}} \underset{\alpha, \mu \rightarrow \infty}{\longrightarrow}-i e^{-\eta i \pi \frac{2 \alpha}{\epsilon_{1}}} e^{-\frac{2 \pi \alpha}{\epsilon_{1}}\left(1-\frac{\mu}{2 \alpha}\right)} \underset{\alpha>\mu}{\longrightarrow} 0
$$


and

$$
B_{a a_{++}}^{\eta} \underset{\epsilon_{2} \rightarrow 0}{\longrightarrow} \frac{\Gamma\left[\frac{2 a}{\epsilon_{1}}\right] \Gamma\left[\frac{2 a}{\epsilon_{1}}\right]}{\Gamma\left[\frac{1}{2}+\frac{2 a-m}{\epsilon_{1}}\right] \Gamma\left[\frac{1}{2}+\frac{2 a+m}{\epsilon_{1}}\right]} e^{\left(\frac{1}{2}+\frac{m}{\epsilon_{1}}\right) \log \frac{2 a}{\epsilon_{1}}} e^{\left(\frac{1}{2}-\frac{m}{\epsilon_{1}}\right) \log \frac{2 a}{\epsilon_{1}}} \underset{|a| \rightarrow \infty}{\longrightarrow} 1 .
$$

In the limit we are considering, we thus obtain the following relation between conformal blocks:

$$
Z\left(a, a_{+} ; z-\tau\right) \sim Z\left(a_{+}, a_{++} ; z\right) .
$$

Defining $Z(a ; z):=Z\left(a, a_{+} ; z\right)$, dividing the above equation by $Z(a ; z)$ on both sides, and making the semiclassical ansatz $Z=\exp \frac{1}{\epsilon_{1} \epsilon_{2}} \mathcal{F}(a)+\frac{1}{\epsilon_{1}} \mathcal{W}(z ; a)$, we arrive at

$$
\begin{aligned}
\lim _{\epsilon_{2} \rightarrow 0} \log \frac{Z(a ; z-\tau)}{Z(a ; z)} & =\frac{1}{\epsilon_{1}}(\mathcal{W}(z-\tau)-\mathcal{W}(z)) \\
& =\lim _{\epsilon_{2} \rightarrow 0} \log \frac{Z\left(a_{+} ; z\right)}{Z(a ; z)} \\
& =\lim _{\epsilon_{2} \rightarrow 0} \frac{1}{\epsilon_{1} \epsilon_{2}}\left(\mathcal{F}\left(a+\frac{\epsilon_{2}}{2}\right)-\mathcal{F}(a)\right) \\
& =\frac{1}{2 \epsilon_{1}} \partial_{a} \mathcal{F}(a),
\end{aligned}
$$

thus reproducing the relation (3.38).

A related line of reasoning, invoking Verlinde operators, appears in [14].

\subsection{The semi-classical S-move kernel}

The S-move kernel relates the one-point conformal blocks with Teichmüller parameter $\tau$ and $-1 / \tau$. It is naturally defined in conventions in which the three-point function contribution in (2.1) is absorbed in the conformal blocks. Denoting the rescaled one-point blocks as $F_{h_{m}}^{h(p)}$, the one-point function is given by $[42,43]$

$$
\left\langle V_{h_{m}}\right\rangle_{\tau}=\int_{0}^{\infty} d p \mu(p) F_{h_{m}}^{h(p)}(\tau) \bar{F}_{h_{m}}^{h(p)}(\tau)
$$

where the weight $h(p)$ is parametrized as $h(p)=\left(\frac{Q}{2}+i p\right)\left(\frac{Q}{2}-i p\right)$ and $\mu(p)$ is the measure factor

$$
\mu(p)=4 \sinh 2 \pi p b \sinh 2 \pi b^{-1} p .
$$

The integral kernel implementing the S-transformation for the block $F_{h_{m}}^{h(p)}(\tau)$ via

$$
F_{h_{m}}^{h\left(p_{2}\right)}(\tau)=\int_{0}^{\infty} d p_{1} \mu\left(p_{1}\right) S_{p_{2} p_{1}}\left(p_{m}\right) F_{h_{m}}^{h\left(p_{1}\right)}\left(-\frac{1}{\tau}\right)
$$

is given by [42]

$$
S_{p_{2} p_{1}}\left(p_{m}\right)=\frac{2^{\frac{3}{2}}}{s_{b}\left(p_{m}\right)} \int_{\mathbb{R}} d r \prod_{\epsilon= \pm} \frac{s_{b}\left(p_{1}+\frac{1}{2}\left(p_{m}+i \frac{Q}{2}\right)+\epsilon r\right)}{s_{b}\left(p_{1}-\frac{1}{2}\left(p_{m}+i \frac{Q}{2}\right)+\epsilon r\right)} e^{4 \pi i p_{2} r},
$$


where the function $s_{b}$ is defined by

$$
\log s_{b}(x)=\frac{1}{i} \int_{0}^{\infty} \frac{d t}{t}\left(\frac{\sin 2 x t}{2 \sinh b t \sinh b^{-1} t}-\frac{x}{t}\right) .
$$

The kernel is invariant under $p_{1} \rightarrow-p_{1}$ as well as $p_{2} \rightarrow-p_{2}$, the latter due to the functional relation

$$
s_{b}(x) s_{b}(-x)=1 .
$$

We would like to compare the exact result (4.24) to the transformation properties of the conformal block $\mathcal{F}$ that we derived in equation (3.58). The identification of parameters ${ }^{2}$

$$
\begin{aligned}
& p_{1}=-i \frac{a_{1}}{\sqrt{\epsilon_{1} \epsilon_{2}}}, \\
& p_{2}=-i \frac{a_{2}}{\sqrt{\epsilon_{1} \epsilon_{2}}}, \\
& p_{m}=-i \frac{m}{\sqrt{\epsilon_{1} \epsilon_{2}}},
\end{aligned}
$$

shows that the $\epsilon_{2} \rightarrow 0$ limit corresponds to the limit in which all momenta are taken large. In this limit, the shift relation

$$
s_{b}(x-i b)=2 \cosh \pi b x s_{b}(x)
$$

gives rise to a first order differential equation for the function $\log s_{b}$, which we can integrate using one special value (e.g. $s_{b}(0)=1$ ), giving rise to the approximation

$$
\lim _{b \rightarrow 0} \log s_{b}(x) \approx \frac{i}{b} \int_{0}^{x} d x^{\prime} \log \left(2 \cosh \pi b x^{\prime}\right) .
$$

We obtain an error estimate for this approximation in appendix A. The S-move kernel in the semi-classical $b \rightarrow 0$ limit is thus approximated by

$$
S_{p_{2} p_{1}}\left(p_{m}\right) \approx \frac{2^{\frac{3}{2}}}{s_{b}\left(p_{m}\right)} \int_{\mathbb{R}} d r \exp \left[4 \pi i p_{2} r+\frac{i}{b} \sum_{\delta, \epsilon= \pm} \delta \int_{0}^{p_{1}+\frac{\delta}{2}\left(p_{m}+i \frac{Q}{2}\right)+\epsilon r} \log \left(2 \cosh \pi b y^{\prime}\right) d y^{\prime}\right] .
$$

Introducing the variables $\alpha_{1}=-i a_{1}, \alpha_{2}=-i a_{2}, \mu=-i m$, we obtain upon the substitution $r \rightarrow \sqrt{\epsilon_{1} \epsilon_{2}} r$

$$
\begin{aligned}
& S_{p_{a} p_{b}}\left(p_{e}\right) \approx \\
& \quad \frac{2^{\frac{3}{2}}}{\sqrt{\epsilon_{1} \epsilon_{2}} s_{b}\left(p_{e}\right)} \int_{\mathbb{R}} d r \exp \left[\frac{1}{\epsilon_{2}}\left(\frac{4 \pi i \alpha_{2} r}{\epsilon_{1}}+i \sum_{\delta, \epsilon= \pm} \delta \int_{0}^{\alpha_{1}+\frac{\delta}{2}\left(\mu+i \frac{\epsilon_{1}+\epsilon_{2}}{2}\right)+\epsilon r} \log \left(2 \cosh \frac{\pi y}{\epsilon_{1}}\right) d y\right)\right] .
\end{aligned}
$$

We will evaluate this expression in a saddle point approximation in the limit $\epsilon_{2} \rightarrow 0$. The saddle points of the exponent satisfy the equation [44] (see also [45])

$$
1=e^{\frac{4 \pi \alpha_{2}}{\epsilon_{1}}} \prod_{\delta, \epsilon= \pm}\left[\cosh \left(\frac{\pi}{\epsilon_{1}}\left(\alpha_{1}+\frac{\delta}{2}\left(\mu+i \frac{\epsilon_{1}+\epsilon_{2}}{2}\right)+\epsilon r\right)\right)\right]^{\delta \epsilon} .
$$

\footnotetext{
${ }^{2}$ As the kernel is independent of $\tau$, the relation (4.24) remains valid with $\tau$ replaced by $-1 / \tau$. There is hence no natural distinction between $a$ and $a_{D}$ in this context.
} 
By invoking

$$
\cosh \left(\frac{a+b}{2}+i \frac{\pi}{4}\right) \cosh \left(\frac{b-a}{2}+i \frac{\pi}{4}\right)=\frac{1}{2}(\cosh a+i \sinh b)
$$

this equation can be put in the form [44]

$$
e^{\frac{-4 \pi \alpha_{2}}{\epsilon_{1}}}=\frac{\cosh \frac{2 \pi \alpha_{1}}{\epsilon_{1}}+i \sinh \frac{\pi(2 r+\mu)}{\epsilon_{1}}}{\cosh \frac{2 \pi \alpha_{1}}{\epsilon_{1}}-i \sinh \frac{\pi(2 r-\mu)}{\epsilon_{1}}},
$$

yielding

$$
\cosh \frac{2 \pi r}{\epsilon_{1}}= \pm i \sinh \frac{\pi\left(2 \alpha_{1} \mp \mu\right)}{\epsilon_{1}}+O\left(e^{\frac{-4 \pi\left|\operatorname{Re} \alpha_{2}\right|}{\epsilon_{1}}}\right) \text { for } \operatorname{Re} \alpha_{2} \rightarrow \pm \infty
$$

and thus

$$
\pm r=\alpha_{1} \mp \frac{1}{2}\left(\mu-i \frac{\epsilon_{1}}{2}\right)+i k \epsilon_{1}+O\left(e^{\frac{-4 \pi\left|\operatorname{Re} \alpha_{2}\right|}{\epsilon_{1}}}\right), \quad k \in \mathbb{Z}, \quad \text { for } \operatorname{Re} \alpha_{2} \rightarrow \pm \infty
$$

where the \pm on the left hand side in the last equation is not correlated with the sign of $\operatorname{Re} \alpha_{2}$. Let us consider the four saddle points closest to the integration path of $r$, which runs along the real axis. Of these, two are zeros of the integrand of (4.25), hence do not correspond to maxima of the real part of the exponential in (4.33). The other two lie on poles of the integrand of (4.25). The integrand evaluated at these yields ${ }^{3}$

$$
\begin{aligned}
& \mu\left(p_{1}\right) S_{p_{2} p_{1}}\left(p_{m}\right) \approx\left(e^{\frac{2 \pi \alpha_{1}}{\epsilon_{2}}}-e^{-\frac{2 \pi \alpha_{1}}{\epsilon_{2}}}\right) \frac{s_{b}\left(\frac{ \pm 2 \alpha_{1}+i \frac{\epsilon_{1}}{2}}{\sqrt{\epsilon_{1} \epsilon_{2}}}\right)}{s_{b}\left(\frac{ \pm 2 \alpha_{1}-\mu}{\sqrt{\epsilon_{1} \epsilon_{2}}}\right)} \cosh \frac{4 \pi i \alpha_{2}\left(\alpha_{1} \mp \frac{1}{2}\left(\mu-i \frac{\epsilon_{1}}{2}\right)\right)}{\epsilon_{1} \epsilon_{2}} \\
& \text { for } \operatorname{Re} \alpha_{2} \rightarrow \pm \infty \text {. }
\end{aligned}
$$

Using this approximation of the S-kernel, a saddle point approximation of the integral (4.24) over $p_{1}$ yields

$$
\partial_{a_{1}} \mathcal{F}_{r}\left(a_{1},-\frac{1}{\tau}\right) \approx \mp 4 \pi i a_{2} \text { for } \operatorname{Re} \alpha_{2} \rightarrow \pm \infty
$$

where $\mathcal{F}_{r}$ denotes the amplitude associated to the rescaled conformal block $F_{h_{m}}^{h(p)}$ introduced above. To leading order, recalling $\mathcal{F}_{0}\left(a_{1}, \tau\right) \approx-2 \pi i a_{1}^{2} \tau$, the relation (4.40) evaluates to

$$
-a_{1} \frac{1}{\tau}= \pm a_{2} \quad \text { for } \operatorname{Re} \alpha_{2} \rightarrow \pm \infty
$$

\footnotetext{
${ }^{3}$ We are shifting the integration contour to run through the saddle points. As these coincide with the poles of the integrand to $O\left(e^{\frac{-4 \pi\left|\operatorname{Re} \alpha_{D}\right|}{\epsilon_{1}}}\right)$, we evaluate the principal value contribution to the integral around these poles. Note that whether the integration path runs above or below the pole is irrelevant for our computation, as the difference between the two is cancelled in relating the integral on the shifted contour to the original integral.
} 
Given the integration region $i \mathbb{R}^{+}$for $a_{1}$, the saddle point which contributes hence depends on the $\operatorname{sign}$ of $\operatorname{Re} \tau$. For $\operatorname{sign}(\operatorname{Re} \tau)= \pm 1$, we obtain

$$
\begin{aligned}
\mathcal{F}_{r}\left(\tau, a_{2}\right) & \approx \pm 4 \pi i \alpha_{1} \alpha_{2}+2 \pi i\left(\alpha_{1}+\alpha_{2}\right)\left(\mu-i \frac{\epsilon_{1}}{2}\right) \pm \frac{\pi i}{2}\left(\mu^{2}+\frac{\epsilon_{1}^{2}}{4}\right)+\mathcal{F}_{r}\left(-\frac{1}{\tau}, a_{1}\right) \\
& =\mp 4 \pi i a_{1} a_{2}-2 \pi i\left(a_{1}+a_{2}\right)\left(m+\frac{\epsilon_{1}}{2}\right) \mp \frac{\pi i}{2}\left(m^{2}-\frac{\epsilon_{1}^{2}}{4}\right)+\mathcal{F}_{r}\left(-\frac{1}{\tau}, a_{1}\right)
\end{aligned}
$$

where we have used

$$
s_{b}(y) \approx e^{ \pm \frac{\pi i}{2}\left(y^{2}+\frac{1}{12 b^{2}}\right)} \quad \text { for } \operatorname{Re}(y) \rightarrow \pm \infty,
$$

see appendix A. Matching to (3.58) requires the identification $a_{2}=a, a_{1}=-a_{D}$ at $\operatorname{sign}(\operatorname{Re} \tau)=1$. The terms linear in $a_{i}$ in the exponential on the right hand side of (4.42) cancel the rescaling of the conformal blocks. While the dependence on the sign of $\operatorname{Re} \tau$ is unusual, note that choosing the fundamental domain of $\tau$ such that this sign is fixed, it is flipped by $\tau \rightarrow-\frac{1}{\tau}$. It can hence serve to distinguish between electric and magnetic variables, which is indeed the role it is playing in equations (4.40) and (4.42). Recall that already in the derivation of (3.58), the argument $-a_{D}$ in $\mathcal{F}\left(-\frac{1}{\tau},-a_{D}\right)$ appeared in the Legendre transform. As $\mathcal{F}$, in contrast to $\mathcal{F}_{r}$, is an even function in this argument, this distinction was not relevant there. We have thus reproduced the Legendre transform relating $\mathcal{F}$ at $\tau$ and $-\frac{1}{\tau}$ from the S-kernel.

\section{Conclusions}

We have seen how $\epsilon_{1}$-deformed Seiberg-Witten relations of $\mathcal{N}=2^{*}$ gauge theory arise naturally within conformal field theory in the context of the $2 \mathrm{~d} / 4 \mathrm{~d}$ correspondence. In particular, we obtained an $\epsilon_{1}$-deformed Seiberg-Witten differential whose $B$-period evaluated on the classical Seiberg-Witten curve gives rise to the derivative of the deformed prepotential. These tools allowed us to prove quasi-modularity of the coefficients of the prepotential from first principles. In the process, we provided a proof of the Matone relation for $\mathcal{N}=2^{*}$ theory. We also demonstrated how the deformed relations can be extracted from the semi-classics of exact conformal field theory quantities.

An important problem for future study is moving beyond leading order in the deformation parameter $\epsilon_{2}$. Aside from recovering all amplitudes $F^{(n, g)}$ from within conformal field theory, it would be important to understand what further modification of the SeibergWitten data is necessary to incorporate these additional corrections. To lift the analysis from gauge theory geometrically engineered within string theory to the topological string proper, it would be interesting to formulate and study a $q$-deformed version of the null vector decoupling equations. Finally, the exact results in conformal field theory which complete relations among the $F^{(n, g)}$ non-perturbatively beg to be interpreted from a gauge theory/topological string theory perspective. 


\section{Acknowledgments}

We would like to thank Francisco Morales and Jörg Teschner for useful conversations. Our work is supported in part by ANR-grant ANR-13-BS05-0001.

\section{A The function $s_{b}$}

The function $s_{b}$ has the integral representation

$$
\log s_{b}(x)=\frac{1}{i} \int_{0}^{\infty} \frac{d t}{t}\left(\frac{\sin 2 x t}{2 \sinh b t \sinh b^{-1} t}-\frac{x}{t}\right) .
$$

We can evaluate the $x$-derivative of this integral by the method of residues:

$$
\begin{aligned}
\frac{d}{d x} \log s_{b}(x) & =\frac{1}{i} \int_{0}^{\infty} d t\left(\frac{\cos 2 x t}{\sinh b t \sinh b^{-1} t}-\frac{1}{t^{2}}\right) \\
& =\frac{1}{i} \mathrm{P} \int_{-\infty}^{\infty} d t\left(\frac{e^{2 i x t}}{2 \sinh b t \sinh b^{-1} t}-\frac{1}{2 t^{2}}\right) \\
& =\frac{1}{i}\left(\int_{\infty}-\int t\left(\frac{e^{2 i x t}}{2 \sinh b t \sinh b^{-1} t}-\frac{1}{2 t^{2}}\right),\right.
\end{aligned}
$$

where we have assumed $\operatorname{Re}(x)>0$ in the last line (else, we close the contour to the bottom). The poles of the integrand lie at $t=i \pi m / b$ and $t=i \pi n b, m, n \in \mathbb{Z}$, with

$$
\begin{aligned}
\operatorname{Res}_{t=i \pi m / b}\left(\frac{e^{2 i x t}}{2 \sinh b t \sinh b^{-1} t}-\frac{1}{2 t^{2}}\right) & =(-1)^{m} \frac{e^{-2 \pi x m / b}}{2 b \sinh \frac{i \pi m}{b^{2}}}, \\
\operatorname{Res}_{t=i \pi n b}\left(\frac{e^{2 i x t}}{2 \sinh b t \sinh b^{-1} t}-\frac{1}{2 t^{2}}\right) & =(-1)^{n} \frac{b e^{-2 \pi x n b}}{2 \sinh i \pi n b^{2}},
\end{aligned}
$$

for $m, n \neq 0$, and

$$
\operatorname{Res}_{t=0}\left(\frac{e^{2 i x t}}{2 \sinh b t \sinh b^{-1} t}-\frac{1}{2 t^{2}}\right)=i x
$$

Thus,

$$
\frac{d}{d x} \log s_{b}(x)=\frac{\pi}{i}\left(-x+\frac{1}{b} \sum_{m=1}^{\infty}(-1)^{m} \frac{e^{-2 \pi x m / b}}{\sin \frac{\pi m}{b^{2}}}+b \sum_{n=1}^{\infty}(-1)^{n} \frac{e^{-2 \pi x n b}}{\sin \pi n b^{2}}\right) .
$$

To take the semi-classical limit, we drop the first sum, and approximate the sine-function in the second,

$$
\begin{aligned}
\frac{d}{d x} \log s_{b}(x) & =\pi i x-i \pi b \sum_{n=1}^{\infty}(-1)^{n} \frac{e^{-2 \pi x n b}}{\pi n b^{2}}+O(b) \\
& =\frac{i}{b} \log (2 \cosh \pi b x)+O(b) .
\end{aligned}
$$

Integrating and imposing the boundary condition $s_{b}(0)=1$ then yields

$$
\log s_{b}(x)=\frac{i}{b} \int_{0}^{x} d x^{\prime} \log \left(2 \cosh \pi b x^{\prime}\right)+O(b) .
$$




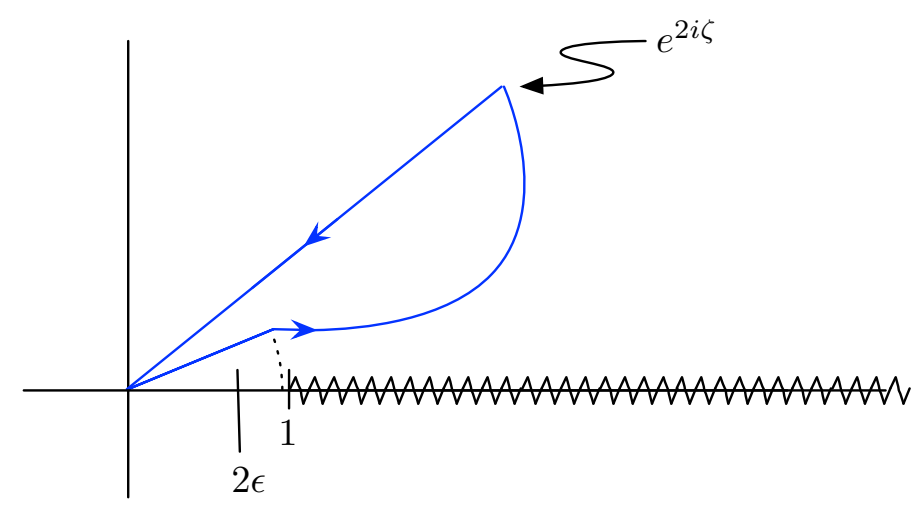

Figure 1. Integration contour.

To obtain the $\operatorname{Re}(x) \rightarrow \pm \infty$ behavior of $s_{b}$, we can relate the right hand side of this approximation to the Lobachevsky function. Defining $S_{b}(x)=s_{b}(y)$ with $x=i y+\frac{Q}{2}$, we obtain

$$
\log S_{b}(x) \approx \frac{1}{b} \int_{Q / 2}^{x} d x^{\prime} \log \left(2 \sin \pi b x^{\prime}\right) \approx \frac{1}{\pi b^{2}} \int_{\frac{\pi}{2}}^{\pi b x} d y \log (2 \sin y) .
$$

The integral on the right hand side can be related to the dilogarithm function [46]. This has the following integral definition:

$$
\operatorname{Li}_{2}(z)=\int_{0}^{z} \log (1-w) \frac{d w}{w}
$$

for $|z| \leq 1$. We choose the branchcut of the logarithm such that the function is analytic away from the semi-axis $[1, \infty)$. Note that for $w=e^{2 i \xi},-\frac{\pi}{2}<\operatorname{Re}(\xi)<\frac{\pi}{2}$,

$$
\log (1-w) \frac{d w}{w}=\log \left(e^{i \xi}\left(e^{-i \xi}-e^{i \xi}\right)\right) 2 i d \xi=(i \xi+\log (-i)+\log (2 \sin \xi)) 2 i d \xi .
$$

Choose the integration path depicted in the figure. Then for $e^{2 i \zeta} \notin[1, \infty)$,

$$
\begin{aligned}
\operatorname{Li}_{2}\left(e^{2 i \zeta}\right)-\operatorname{Li}_{2}\left(e^{2 i \epsilon}\right) & =-\int_{\epsilon}^{\zeta}(i \xi+\log (-i)+\log (2 \sin \xi)) 2 i d \xi \\
& =-2 i \int_{\epsilon}^{\zeta} \log (2 \sin \xi) d \xi-\pi(\zeta-\epsilon)+\zeta^{2}-\epsilon^{2}
\end{aligned}
$$

Taking the $\epsilon \rightarrow 0$ limit yields

$$
\int_{0}^{\zeta} \log (2 \sin \xi) d \xi=\frac{i}{2}\left(\operatorname{Li}_{2}\left(e^{2 i \zeta}\right)-\frac{\pi^{2}}{6}+\zeta(\pi-\zeta)\right)
$$

where we have used $\operatorname{Li}_{2}(1)=\frac{\pi^{2}}{6}$. We are interested in the $\operatorname{Im} \zeta \rightarrow \pm \infty$ limits of this expression. The $\operatorname{Im} \zeta \rightarrow+\infty$ limit follows immediately from $\operatorname{Li}_{2}(0)=0$,

$$
\int_{0}^{\zeta} \log (2 \sin \xi) d \xi \underset{\operatorname{Im} \zeta \rightarrow+\infty}{\longrightarrow}-\frac{i}{2}\left(\frac{\pi^{2}}{6}-\zeta(\pi-\zeta)\right) .
$$


For the $\operatorname{limit} \operatorname{Im} \zeta \rightarrow-\infty$, note that

$$
\operatorname{Li}_{2}(z)+\operatorname{Li}_{2}(1 / z)=-\frac{\pi^{2}}{6}-\frac{1}{2} \log ^{2}(-z)
$$

hence

$$
\lim _{\operatorname{Im} \zeta \rightarrow \infty} \operatorname{Li}_{2}\left(e^{2 i \zeta}\right)=-\frac{\pi^{2}}{6}-\frac{1}{2} \log ^{2} e^{-i \pi+2 i \zeta}=\frac{2 \pi^{2}}{6}-2 \zeta(\pi-\zeta) .
$$

To arrive at this result, remember that the branchcut of $\log z$ is chosen along the negative real axis, and that $-\frac{\pi}{2}<\operatorname{Re}(\xi)<\frac{\pi}{2}$. Thus,

$$
\int_{0}^{\zeta} \log (2 \sin \xi) d \xi \underset{\operatorname{Im} \zeta \rightarrow-\infty}{\longrightarrow} \frac{i}{2}\left(\frac{\pi^{2}}{6}-\zeta(\pi-\zeta)\right) .
$$

Substituting this result into (A.12) and using

$$
\int_{0}^{\frac{\pi}{2}} d y \log (2 \sin y)=0
$$

yields

$$
\log S_{b}(x) \underset{\operatorname{Im} x \rightarrow \pm \infty}{\stackrel{b \rightarrow 0}{\longrightarrow}} \mp \frac{\pi i}{2}\left(x\left(x-\frac{1}{b}\right)+\frac{1}{6 b^{2}}\right)
$$

and thus

$$
\log s_{b}(x) \underset{\operatorname{Re} x \rightarrow \pm \infty}{\stackrel{b \rightarrow 0}{\longrightarrow}} \pm \frac{\pi i}{2}\left(x^{2}+\frac{1}{12 b^{2}}\right) .
$$

Open Access. This article is distributed under the terms of the Creative Commons Attribution License (CC-BY 4.0), which permits any use, distribution and reproduction in any medium, provided the original author(s) and source are credited.

\section{References}

[1] L.F. Alday, D. Gaiotto and Y. Tachikawa, Liouville correlation functions from four-dimensional gauge theories, Lett. Math. Phys. 91 (2010) 167 [arXiv:0906.3219] [INSPIRE].

[2] M. Bershadsky, S. Cecotti, H. Ooguri and C. Vafa, Kodaira-Spencer theory of gravity and exact results for quantum string amplitudes, Commun. Math. Phys. 165 (1994) 311 [hep-th/9309140] [INSPIRE].

[3] D. Krefl and J. Walcher, Extended holomorphic anomaly in gauge theory, Lett. Math. Phys. 95 (2011) 67 [arXiv:1007.0263] [InSPIRE].

[4] M.-x. Huang and A. Klemm, Direct integration for general $\Omega$ backgrounds, Adv. Theor. Math. Phys. 16 (2012) 805 [arXiv:1009.1126] [INSPIRE].

[5] M.-x. Huang, A.-K. Kashani-Poor and A. Klemm, The $\Omega$ deformed B-model for rigid $\mathcal{N}=2$ theories, Ann. Henri Poincaré 14 (2013) 425 [arXiv:1109.5728] [InSPIRE].

[6] N.A. Nekrasov, Seiberg-Witten prepotential from instanton counting, Adv. Theor. Math. Phys. 7 (2004) 831 [hep-th/0206161] [INSPIRE]. 
[7] A.-K. Kashani-Poor and J. Troost, The toroidal block and the genus expansion, JHEP 03 (2013) 133 [arXiv: 1212.0722] [INSPIRE].

[8] A.-K. Kashani-Poor and J. Troost, Transformations of spherical blocks, JHEP 10 (2013) 009 [arXiv: 1305.7408] [INSPIRE].

[9] N. Seiberg and E. Witten, Electric-magnetic duality, monopole condensation and confinement in $N=2$ supersymmetric Yang-Mills theory, Nucl. Phys. B 426 (1994) 19 [Erratum ibid. B 430 (1994) 485] [hep-th/9407087] [INSPIRE].

[10] N. Seiberg and E. Witten, Monopoles, duality and chiral symmetry breaking in $N=2$ supersymmetric QCD, Nucl. Phys. B 431 (1994) 484 [hep-th/9408099] [INSPIRE].

[11] N.A. Nekrasov and S.L. Shatashvili, Quantization of integrable systems and four dimensional gauge theories, arXiv:0908.4052 [INSPIRE].

[12] M. Matone, Instantons and recursion relations in $N=2$ SUSY gauge theory, Phys. Lett. B 357 (1995) 342 [hep-th/9506102] [INSPIRE].

[13] R. Flume, F. Fucito, J.F. Morales and R. Poghossian, Matone's relation in the presence of gravitational couplings, JHEP 04 (2004) 008 [hep-th/0403057] [INSPIRE].

[14] L.F. Alday, D. Gaiotto, S. Gukov, Y. Tachikawa and H. Verlinde, Loop and surface operators in $N=2$ gauge theory and Liouville modular geometry, JHEP 01 (2010) 113 [arXiv: 0909.0945] [INSPIRE].

[15] A. Iqbal, C. Kozcaz and C. Vafa, The refined topological vertex, JHEP 10 (2009) 069 [hep-th/0701156] [INSPIRE].

[16] V.A. Fateev and A.V. Litvinov, Multipoint correlation functions in Liouville field theory and minimal Liouville gravity, Theor. Math. Phys. 154 (2008) 454 [arXiv:0707.1664] [INSPIRE].

[17] K. Maruyoshi and M. Taki, Deformed prepotential, quantum integrable system and Liouville field theory, Nucl. Phys. B 841 (2010) 388 [arXiv: 1006.4505] [INSPIRE].

[18] A. Marshakov, A. Mironov and A. Morozov, On AGT relations with surface operator insertion and stationary limit of beta-ensembles, J. Geom. Phys. 61 (2011) 1203 [arXiv: 1011.4491] [INSPIRE].

[19] S.D. Mathur, S. Mukhi and A. Sen, Correlators of primary fields in the $\mathrm{SU}(2) W Z W$ theory on Riemann surfaces, Nucl. Phys. B 305 (1988) 219 [INSPIRE].

[20] D. Harlow, J. Maltz and E. Witten, Analytic continuation of Liouville theory, JHEP 12 (2011) 071 [arXiv:1108.4417] [INSPIRE].

[21] M. Billó, M. Frau, L. Gallot and A. Lerda, The exact $8 d$ chiral ring from 4 d recursion relations, JHEP 11 (2011) 077 [arXiv:1107.3691] [INSPIRE].

[22] M. Billó, M. Frau, L. Gallot, A. Lerda and I. Pesando, Deformed $N=2$ theories, generalized recursion relations and S-duality, JHEP 04 (2013) 039 [arXiv: 1302.0686] [INSPIRE].

[23] R. Donagi and E. Witten, Supersymmetric Yang-Mills theory and integrable systems, Nucl. Phys. B 460 (1996) 299 [hep-th/9510101] [INSPIRE].

[24] E. Witten, Solutions of four-dimensional field theories via M-theory, Nucl. Phys. B 500 (1997) 3 [hep-th/9703166] [INSPIRE].

[25] D. Gaiotto, $N=2$ dualities, JHEP 08 (2012) 034 [arXiv:0904.2715] [INSPIRE]. 
[26] A. Zabrodin and A. Zotov, Quantum Painleve-Calogero correspondence, J. Math. Phys. 53 (2012) 073507 [arXiv:1107.5672] [INSPIRE].

[27] M. Grosset and A. Veselov, Elliptic Faulhaber polynomials and Lamé densities of states, math-ph/0508066.

[28] M. Aganagic, R. Dijkgraaf, A. Klemm, M. Mariño and C. Vafa, Topological strings and integrable hierarchies, Commun. Math. Phys. 261 (2006) 451 [hep-th/0312085] [INSPIRE].

[29] M. Aganagic, M.C.N. Cheng, R. Dijkgraaf, D. Krefl and C. Vafa, Quantum geometry of refined topological strings, JHEP 11 (2012) 019 [arXiv:1105.0630] [INSPIRE].

[30] M.-x. Huang, On gauge theory and topological string in Nekrasov-Shatashvili limit, JHEP 06 (2012) 152 [arXiv: 1205.3652] [INSPIRE].

[31] M.-x. Huang, A. Klemm, J. Reuter and M. Schiereck, Quantum geometry of del Pezzo surfaces in the Nekrasov-Shatashvili limit, arXiv:1401.4723 [INSPIRE].

[32] R. Poghossian, Deforming SW curve, JHEP 04 (2011) 033 [arXiv: 1006.4822] [INSPIRE].

[33] F. Fucito, J.F. Morales, D.R. Pacifici and R. Poghossian, Gauge theories on $\Omega$-backgrounds from non commutative Seiberg-Witten curves, JHEP 05 (2011) 098 [arXiv:1103.4495] [INSPIRE].

[34] A. Mironov and A. Morozov, Nekrasov functions and exact Bohr-Zommerfeld integrals, JHEP 04 (2010) 040 [arXiv:0910.5670] [INSPIRE].

[35] G. Bonelli, K. Maruyoshi and A. Tanzini, Quantum Hitchin systems via $\beta$-deformed matrix models, arXiv:1104.4016 [INSPIRE].

[36] J.-E. Bourgine, Large- $N$ limit of $\beta$-ensembles and deformed Seiberg-Witten relations, JHEP 08 (2012) 046 [arXiv:1206.1696] [INSPIRE].

[37] J.-E. Bourgine, Large- $N$ techniques for Nekrasov partition functions and AGT conjecture, JHEP 05 (2013) 047 [arXiv: 1212.4972] [INSPIRE].

[38] T. Eguchi and H. Ooguri, Conformal and current algebras on general Riemann surface, Nucl. Phys. B 282 (1987) 308 [inSPIRE].

[39] D. Galakhov, A. Mironov and A. Morozov, S-duality as a $\beta$-deformed Fourier transform, JHEP 08 (2012) 067 [arXiv: 1205.4998] [INSPIRE].

[40] N. Nemkov, S-duality as Fourier transform for arbitrary $\epsilon_{1}, \epsilon_{2}$, arXiv:1307.0773 [INSPIRE].

[41] G.W. Moore and N. Seiberg, Classical and quantum conformal field theory, Commun. Math. Phys. 123 (1989) 177 [INSPIRE].

[42] J. Teschner, From Liouville theory to the quantum geometry of Riemann surfaces, hep-th/0308031 [INSPIRE].

[43] G. Vartanov and J. Teschner, Supersymmetric gauge theories, quantization of moduli spaces of flat connections and conformal field theory, arXiv:1302.3778 [INSPIRE].

[44] L. Hadasz and Z. Jaskolski, Semiclassical limit of the FZZT Liouville theory, Nucl. Phys. B 757 (2006) 233 [hep-th/0603164] [INSPIRE].

[45] T. Dimofte and S. Gukov, Chern-Simons theory and S-duality, JHEP 05 (2013) 109 [arXiv:1106.4550] [INSPIRE].

[46] J.G. Ratcliffe, Foundations of hyperbolic manifolds, vol. 149 of Graduate Texts in Mathematics, second edition, Springer, New York U.S.A. (2006). 